\title{
Data Integration in Big Data Environment
}

\author{
B. Arputhamary and L. Arockiam
}

\begin{abstract}
Data Integration is the process of transferring the data in source format into the destination format. Many data warehousing and data management approaches has been supported by integration tools for data migration and transportation by using Extract-Transform-Load (ETL) approach. These tools are widely fit for handling large volumes of data and not flexible to handle semi or unstructured data. To overcome these challenges in big data world, programmatically driven parallel techniques such as map-reduce models were introduced. Data Integration as a process is highly cumbersome and iterative especially to add new data sources. The process of adding these new data sources are time consuming which results in delay, loss of data and irrelevance of the data and improper utilization of useful information. Traditionally waterfall approach is used in EDW (Enterprise Data Warehouse), where one cannot move to the next phase before completing the earlier one. This approach has its merits to ensure the right data sources are picked and right data integration processes are developed to sustain the usefulness of EDW. In big data environment, the situation is completely different. Therefore the traditional approaches of integration are inefficient in handling the current situation. So people are expected to do something regarding this issue. In this paper the importance of data integration in Big Data world are identified and the open problems of Big Data Integration are outlined to proceed future research in Big Data environment.
\end{abstract}

Keywords--- Big Data, Bid Data Analytics, Data Integration

\section{INTRODUCTION}

$\mathrm{T}$ HE term 'Big Data' basically derived from the big or large volume of data. There is no proper definition for Big Data. It was defined as a situation where the volume, velocity and variety of data exceed an organization's storage or compute capacity for accurate and timely decision making. Storage of these big data can be done by introducing multiple data centers, where as utilizing these data in an effective manner is a tedious one which is to be considered carefully. Today, organizations invest more in data manipulation and most of the time the stored data are unused and they are not retrieved and utilized in a proper way. Enterprises and

B. Arputhamary, Assistant Professor, Department of Computer Applications, Bishop Heber College, Trichy, India, E-mail: arputhambaskaran@rediffmail.com

Dr. L. Arockiam, Associate Professor, Department of Computer Science, St.Joseph's College, Trichy, India, E-mail: Larockiam@yahoo.co.in

DOI: 10.9756/BIJDM.8001 organizations are spending more in data processing. Actually big data is not a problem whereas it is a big asset to the organizations. Data are from different sources and integrating such data is very important and available data warehousing tools are used for doing integration. But in big data environment data from different sources are of different formats and existing data warehousing techniques are inefficient to handle such situation. This paper aims at presenting the issues related to data integration in big data environment and techniques available for Big Data Integration.

\section{BACKGROUND}

Xin Luna Dong et al.,[9], aims at providing the progress by data integration community on the topics of schema mapping, record linkage and data fusion. The authors have tried to identify the challenges of these areas in big data environment. This paper describes how Big Data Integration differs from data integration with respect to 5 V's. Solution for achieving schema mapping is suggested as Hadoop which is in contrast to the traditional approach define the schema after the data has been stored. For record linkage, the authors have identified that Map Reduce model for handling large volume of data. Clustering techniques were suggested to solve the velocity problems. To address the variety aspect, techniques have been proposed that tag and match free text to structured data. Data fusion is a new field which emerged recently due to veracity. Also this paper is very helpful to find the open problems of data integration in Big Data Environment and the problems are, Integrating Crowd sourcing data, Integrating Data from data markets and providing an exploration tool for data sources. Nikhil Debbarma et al.,[10] made study on the issues related to Data Warehouse in the quality perspective. Data quality plays an important role in Data Warehousing and Data Warehouse plays a vital role to extract right information from the right place at the right time with the right cost, in order to take right decision. This paper has outlined the impacts of poor quality data in decision making and the cost of erroneous data. Completeness, Consistency, Validity, Integrity, Conformity, Accuracy are the important parameters to be considered in data quality. The authors have worked towards the steps on data warehouse extraction, transformation and load they have also identified the quality related problems on ETL process. Data Profiling is the mechanism that has been suggested as a future scope in data warehousing to ensure data quality. Jin Zhou et al.,[12] has considered Internet of Things (IOT) to generate large amounts of multisource, heterogeneous, dynamic and sparse data. To acquire an acceptable quality of intelligence, the authors have decided to combine diverse and voluminous data. The authors have proposed multidimensional fusion algorithm for IOT data, 
based on partitioning. To achieve the main objective, three mechanisms such as finding the core attributes, determining global core attributes from the core attributes and finally attribute reduction based on rule extraction are proposed. Mian Wang et al.,[13], proposed a framework for set similarity join. This approach improves the efficiency by reducing candidate pairs and load balance. The authors have proposed an algorithm to reduce the amount of candidate pairs. Multiple filtering principles such as length filter, Prefix filter and Position filter are used. Load balancing techniques are also used to address the skew data and decrease the replication transfer volume. They proposed a filter framework that combines multiple filter algorithms and uses Map Reduce to process similarity join tasks in parallel. Rashmi Ranjan et al.,[20] have proposed a systematic time series based scheme to assess the demand of the product by tracking its behavior and predict the future response of customers depending on the present dataset as well as historical dataset using Hadoop framework and Holt-Winter prediction function in the $\mathrm{R}$ environment. In future by the capturing the sentiments of users about a given product from different social networks, the performance of predictive and sentiment analytics can be improved. The main objective of this research [21] is extracting web content with high volume and heterogeneous information which continues to expand in size and complexity. Existing approaches are proposed based on automatic extraction and hand crafted rule generation. In this research work a hybrid approach is proposed to extract main content from the web pages. Decision tree classification and Naïve Bayes Classification machine learning methods are used for rules generation. The performance of machine learning methods is compared with metrics like precision, recall, fmeasure and accuracy. In this paper the authors Umeshwar Dayal et al.,[22] discussed the objectives of Business Intelligence in Data Integration. The objectives are, reducing the latency, Extracting Information from a wider variety of data sources and extending the serial ETL to more general data flows. The research mainly concentrate on proposing a framework by identifying the current requirements of Data Integration, Limitations in current technologies and research challenges in meeting all these requirements. The authors also identified and outlined the future demand on data integration

flows.

\section{OVERVIEW OF DATA INTEGRATION}

The World Wide Web has rich source of voluminous and heterogeneous information which continuous to expand in size and complexity. Many web pages are unstructured and semi structured. Information extraction is first and an important task in data integration process. Data Extraction takes data from source systems and makes it available to the data warehouse. Main objective of this extraction process is to extract right information from the right place at the right time with the right cost to support right decision. Traditionally, data warehouses were used as multidimensional databases to store large amounts of data. The downsides of the data warehouses are ensuring quality of arriving data. Poor data quality results in poor decision making which ultimately results in poor performance. Completeness, Consistency and Correctness of data supports organizations and is the cornerstone of decision making systems in organizations. Good functioning and efficiency of an organization depends on the quality of the data. Poor data quality decreases customers satisfaction and automatically degrades the performance and decision making ability[4][16].

\section{A. Extract, Transform and Load Process}

ETL comes from Data Warehousing and stands for Extract-Transform-Load. ETL performs the process of loading data from the source system to target system. It is converting data of same type or different types to centralized Data Warehouse which is having standard format for all data[5]-[7].

\section{i. $\quad$ Extract}

The "Extract" task involves gathering data from external sources that needs to be brought to the required systems and databases. The data might be found in stand-alone databases or spreadsheets that aren't integrated with any master database. The data might even be hard-copy or could be data that have been purchased from a data provider. The goal of this task is to understand the format of data, assess the overall quality of the data and to extract the data from its source so that it can be manipulated in next task.

\section{ii. Transform}

In the "transform" step a variety of software tools and even custom programming are used to manipulate the data so that it integrates with data that is already present.

\section{iii. Load}

After the successfully transformation of the source data it is required to physically load it into the target system or database. Before loading the data, it is required to make sure that there is a backup of the current system so that roll back or undo can be initiated in case of failure of the Load process. After loading the data, it's common to run audit reports so that there can be review of the results of the merged databases and systems to make sure the new data hasn't caused any errors. The following diagram describes the general steps in traditional data warehousing process[10].

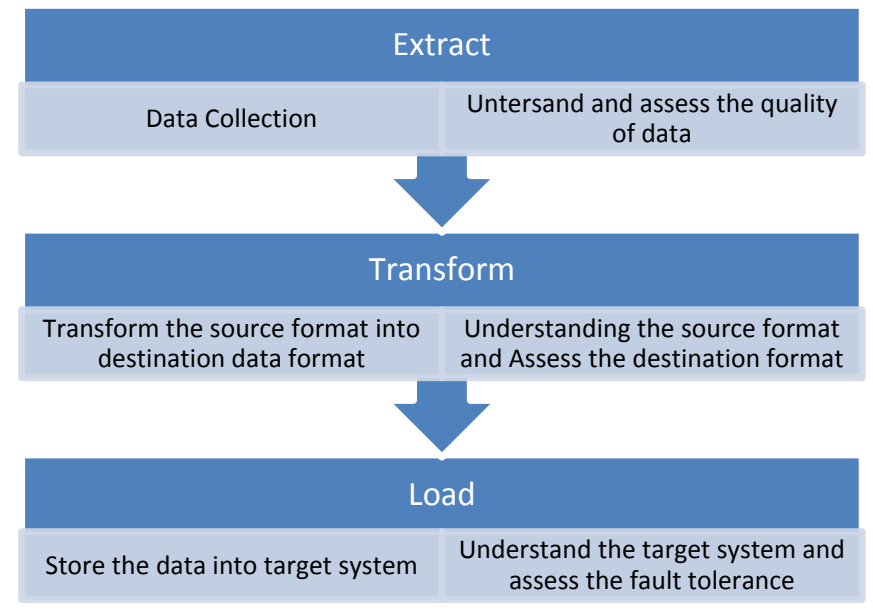

Figure 1: The General Process of ETL 
Table I: Data Quality in ETL

\begin{tabular}{|l|l|l|}
\hline \multicolumn{3}{|c|}{ Issues in Data Quality during data integration process } \\
\hline \multicolumn{1}{|c|}{ Extract } & \multicolumn{1}{|c|}{ Transform } & \multicolumn{1}{c|}{ Load } \\
\hline $\begin{array}{l}\text { Heterogeneous } \\
\text { data sources } \\
\text { having own } \\
\text { storage methods. }\end{array}$ & $\begin{array}{l}\text { Insufficient source } \\
\text { data analysis. }\end{array}$ & $\begin{array}{l}\text { Lack of Periodical } \\
\text { refreshment of integrated } \\
\text { data cause for data } \\
\text { quality problems. } \\
\text { alterations. }\end{array}$ \\
$\begin{array}{l}\text { Imperfect schema } \\
\text { level definition. }\end{array}$ & $\begin{array}{l}\text { Unhandled null mapping of data } \\
\text { leading to data being } \\
\text { values in ETL } \\
\text { eroneous. }\end{array}$ \\
$\begin{array}{l}\text { Imperfect } \\
\text { Instance data. }\end{array}$ & $\begin{array}{l}\text { Misinterpreting / wrong } \\
\text { implementation of the } \\
\text { Slowly Changing } \\
\text { Insufficient } \\
\text { source data } \\
\text { analysis. }\end{array}$ & $\begin{array}{l}\text { conditional } \\
\text { statements force } \\
\text { the valid data to } \\
\text { move to the invalid } \\
\text { tables. }\end{array}$ \\
$\begin{array}{l}\text { Undocumented } \\
\text { alterations. }\end{array}$ & $\begin{array}{l}\text { Lack of error reporting, } \\
\text { validation and metadata } \\
\text { updates in ETL process. } \\
\text { Inappropriate handling of } \\
\text { return strategies during }\end{array}$ \\
\hline
\end{tabular}

\section{QOX METRICS IN DATA INTEGRATION}

The followings are the some of the QOX ( Quality of Service, Quality of Data, Quality of Information etc.,) metrics in data integration[21],[22].

Reliability: The probability that the ETL process will perform its intended operation during specified time period under given conditions. Any reason for not performing the intended operation is considered to be a failure.

Maintainability: The ability of an ETL process to be operated at the design cost and with service level agreements.

Freshness: The ability of the system to provide the desired latency in updating the data warehouse.

Recoverability: The ability to restore an ETL process to the point at which a failure occurred within a specified time window.

Scalability: The ability of an ETL process to handle higher volumes of data.

Availability: The probability that the ETL process is operational during a specific time period.

Flexibility: The ability to accommodate previously unknown, new or changing requirements.

Robustness: The ability of an ETL process to continue operating well or with minimal harm.

Affordability: The ability to maintain or scale the cost of an ETL process appropriately.

Consistency: The extent to which the data populating the data warehouse is correct and complete.

Traceability: the ability of an ETL process to track the lineage of data and data changes.

Auditability: The ability of an ETL process to protect data privacy and security, and to provide data and business rule transparency.

\section{BIG DATA AND ITS CHARACTERISTICS}

Big Data means datasets which could not be captured, managed and processes by general computers within an acceptable scope, Apache Hadoop,2014. According to Gartner[18], Big data is defined as high-volume, high-velocity and high-variety information assets that demand costeffective, innovative forms of information processing for enhanced insight and decision making. The three V's of Big Data are - Volume, Velocity, and Variety. When most people hear the term Big Data, they assume it to be a massive transactional data set. However, volume is only the first dimension of Big Data, and is potentially the least important among all three dimensions [1][15]-[20].

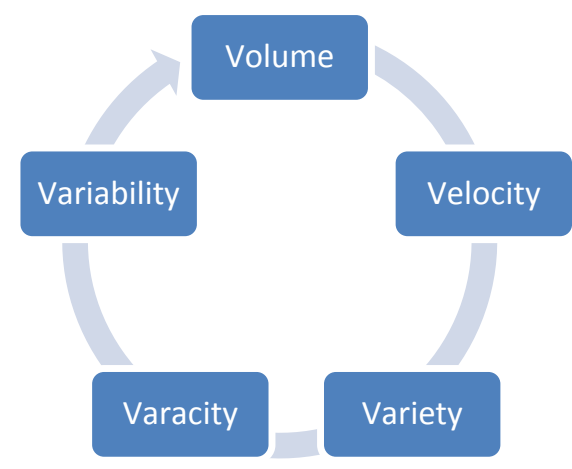

Figure 2: Characteristics of Big Data

- Volume - Volume derives the amount of data from terabytes to Peta bytes.

- Velocity - Velocity represents speed (ie.) Velocity means the rate of change in the data and how fast it must be processed to gain business value.

- Variety - Big Data means much more than traditional RDBMS data. It includes unstructured text, sound and movie files, images, documents, geo-location data, web logs, etc.

Now two V's are added namely Veracity and Variability

- Veracity - Data in doubt or unpredictable data.

- Variability - Semantics or the variability in language [2].

\section{OVERVIEW OF BIG DATA INTEGRATION}

Big data integration (BDI) means linking or fusing large volumes of heterogeneous data from many dynamic data sources. The main differences of BDI from traditional Data Integration are [9],

- The number of data sources even for a single domain has grown to be in the tens of thousands.

- Many of the data sources are dynamic.

- The data sources are extremely heterogeneous in their structure.

- The data sources are of widely differing qualities with significant differences in the coverage, accuracy and timeliness of data provided.

The schema mapping, record linkage and data fusion are challenging areas of data integration in Big Data environment. The following figure describes integration techniques in meeting the big data challenges with respect to the $5 \mathrm{~V}$ 's. 


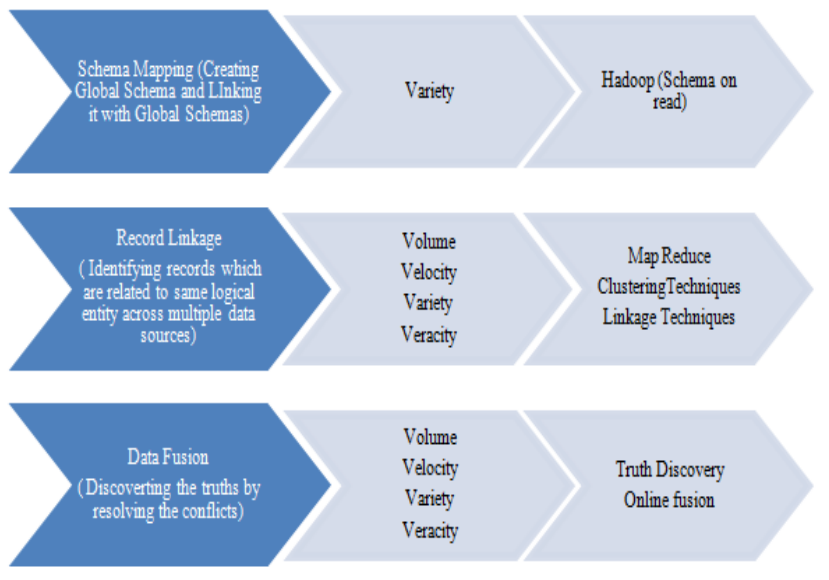

Figure 3: Big Data Integration Challenges and Techniques
The following section describes analysis of different platforms and its advantages in Big Data environment.

\section{Platforms of Big Data ANALYTics}

Big Data analytics means identifying hidden patterns and business information from large amounts of data, to make the business more agile. This data comes from everywhere: social network activity, web server logs, sensors used to gather climate information, stock market data, e-mails, transaction records, web click streams, etc. Analytics is an important task to take right decisions in order to improve the businesses. This section outlines the importance of scalability to extract and analyze data in high volume with heterogeneity. The following table summarizes the platforms for Big Data Analytics under horizontal and vertical scaling [23],[24].

Table II: Big Data Analytics Platforms

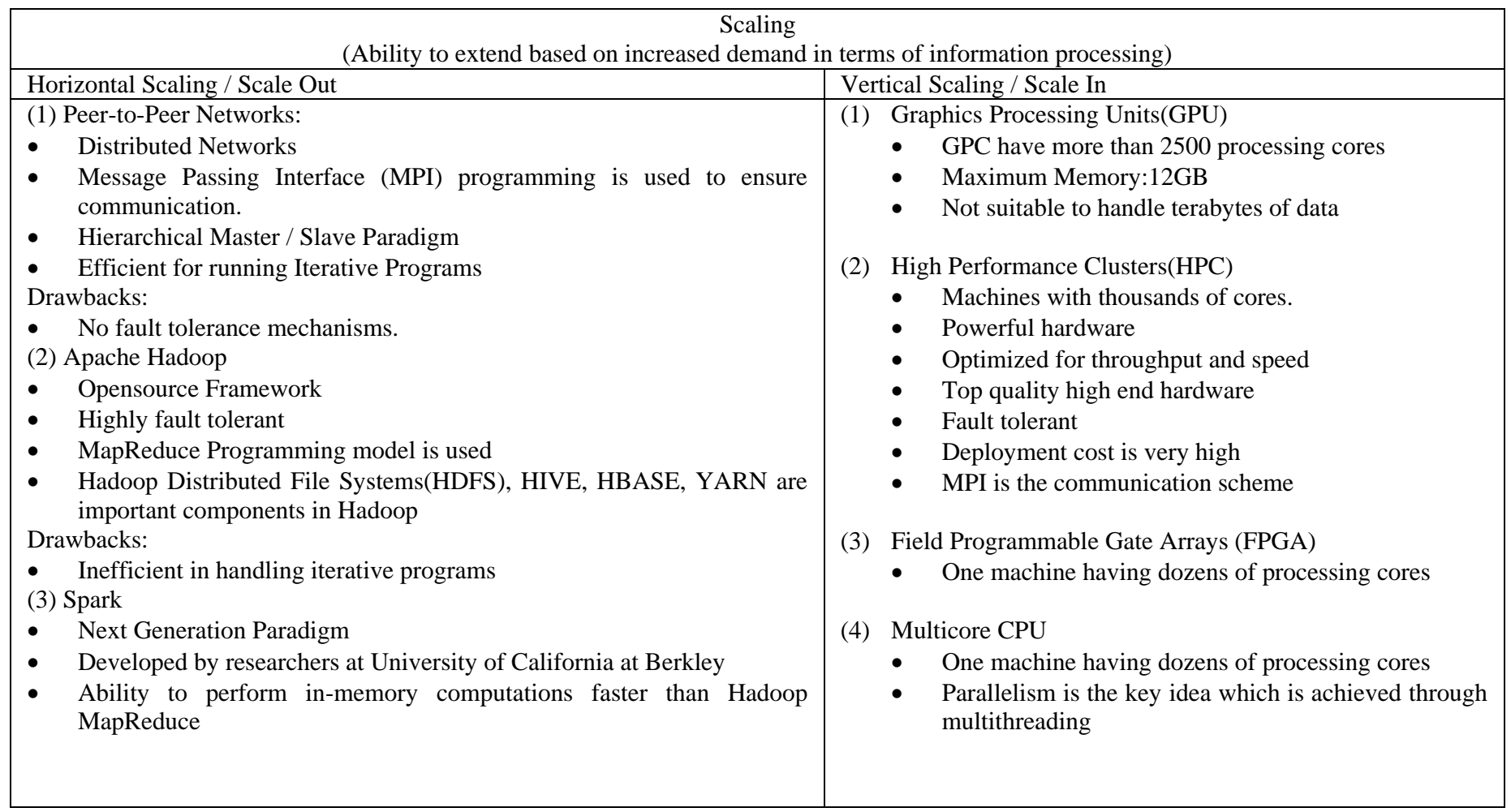

VIII. OPEN ISSUES IN BIG DATA TECHNOLOGY

This section describes the possible research directions of big data[1]-[3],[25].

- Format conversion mechanisms are required due to its heterogeneity

- Improving the transfer efficiency ( data migration process)

- Computing models to be built to improve the real time performance of Big Data

- Mechanisms are required to process high volumes of data with huge velocity and wide variety.

- Big Data Privacy and Data Quality must be improved at security level

- Mechanisms to integrate different data sources

\section{CONCLUSION}

Today data are being generated, collected and analyzed at an unprecedented scale which creates problems in management and manipulation of data. Big Data Integration is the major challenge in Big Data Environment due to its heterogeneity nature. This paper aims at giving the outline of challenges of data integration related to Big Data environment. From the study of Big Data Integration, it is identified as the existing techniques and approaches are inefficient to handle the problems. Therefore a new framework, technique or algorithms can be proposed in future. And also this paper identifies some open problems in big data integration for future research. 


\section{REFERENCES}

[1] Min Chen, Shiwen Mao, Yunhao Liu " Big Data: A Survey", Springer Science + Business Media, New York 2014,DOI 10.1007/s 11036-0130489-0, Springer,2014.

[2] Sachchidanand Singh, Nirmala Singh, "Big Data Analytics", International Conference on Communication, Information \& Computing Technology (ICCICT), Oct 19-20, 2012.

[3] Dilpreet Singh and Chandan K Reddy, "A Survey on Platforms of Big Data Analytics", Journal of Big Data, Springer Open Acess,2014.

[4] Big Data and Enterprise Data: Bridging Two worlds with Oracle Integrator12C(ODI12C), An Oracle White Paper, Sep 2013.

[5] Soumy sen "Integrating XML Data into Multiple ROLAP Data Warehouse Schemas", International Journal of Software Engineering and Application (USEA), Vol 3,No.1, Jan 2012.

[6] Soumy sen "Integrating XML Data into Multiple ROLAP Data Warehouse Schemas", International Journal of Software Engineering and Application (USEA), Vol 3,No.1, Jan 2012.

[7] Rajni Jindal, Shewta Taneja, "Comparative study of Data Warehouse Design Approaches: A Survey", International Journal of Database Management Systems (IJDMS), vol.4, No.1, Feb 2012.

[8] Xiongpai QIN,Huiju WANG, Furong LI, Baoyao ZHOU "Beyond Simple Integration of RDBMS and MapReduce-Paying the Way toward a Unified System for Big Data Analytics: Vision and Progress", 2012 Second International Conference on Cloud and Green Computing, 9780-7695-4864-7/12\$26.00@2012 IEEE, DOI 10.1109/CGC.2012.39.

[9] Xin Luna Dong, Divesh Srivastava, "Big Data Integration" ICDE conference 2013, 978-1-4673-4910-9/13/\$31.00@2013 IEEE.

[10] Nikhil Debbarma, Gautam Nath, Hillo I Das, “ Analysis of Data Quality and Performance Issues in Data Warehousing and Business Intelligence", International Journal of Computer Applications(09758887), Volume 79, No 15, October 2013.

[11] Aisha Naseer, Loredana Laera, Takahide Matsutsuka, "Enterprise BigGraph", $201346^{\text {th }}$ Hawaii International Conference on System Sciences, 1530-1605/12 @ IEEE, DOI 10.1109/ HICSS.2013 .202,2012.

[12] Jin Zhou, Liang Hu, Feng Wang, Huimin Lu and Kuo Zhao, "An Efficient Multidimensional Fusion Algorithm for IOT Data Based On Partitioning", Tsinghua Science and Technology, ISSN 1007-0214 05/10 pp 369-378, Volume 18, Number 4, August 2013.

[13] Mian Wang, "Intelligent Similarly Joins for Big Data Integration, 2013.

[14] Andreas Schultz, "LDIF- A framework for Large-Scale Linked Data Integration" www 2012 Developer Track, Apr 18-20, 2012, Lyon, France.

[15] Gueyoung Jung, Nathan Gnanasambandam, Tridib Mukherjee "Synchronous Parallel Processing of Big-Data Analytics Services to Optimize Performance in Federated Clouds", 2012 IEEE Fifth International Conference on Cloud Computing, 978-0-7695-47558/12\$26.00@2023 IEEE. DOI 10.1109/CLOUD.2012.108.

[16] Steven J. Rysavy, Dennis Bromley, and Valerie Daggett "DIVE: A Graph-Based Visual- Analytics Framework for Big Data" March/April 2014 Published by the IEEE Computer Society 0272-1716/14/\$31.00 (C) 2014 IEEE.

[17] Amgad Madkour, Walid G. Aref, Saleh Basalamah "Knowledge Cubes A Proposal for Scalable and Semantically-Guided Management of Big Data", partially supported by the National Sci- ence Foundation under Grants III-1117766, and IIS-0964639, and IIS-0916614.

[18] Kiyana Zolfaghar, Naren Meadem, Ankur Teredesai, Senjuti Basu Roy, Brian Muckian "Big Data Solutions for Predicting Risk-of-Readmission for Congestive Heart Failure Patients ",2013 IEEE International Conference on Big Data, 978-1-4799-1293-3/13/\$31.00 (C2013 IEEE.

[19] Sandro Fiore, Cosimo Palazzo, Alessandro D’Anca, Ian Foster, Dean N. Williams, Giovanni Aloisio "A big data analytics framework for scientific data management" 2013 IEEE International Conference on Big Data, 978-1-4799-1293-3/13/\$31.00 @2013 IEEE.

[20] Rashmi Ranjan Dhal and B.V.A.N.S.S. Prabhakar Rao, " Shrinking the Uncertainity in ,Online Sales Prediction With Time Series Analysis", ICTACT JOURNAL ON SOFT COMPUTING: SPECIAL ISSUE ON DISTRIBUTED INTELLIGENT SYSTEMS AND APPLICATIONS, OCTOBER 2014, VOLUME: 05, ISSUE: 01,2014.

[21] K.Nethra, J.Anitha, G.Thilagavathi, "Web Content Extraction Using Hybrid Approach", ICTACT JOURNAL ON SOFT COMPUTING, JANUARY 2014, VOLUME: 04, ISSUE: 02, 2014.

[22] Umeshwar Dayal, Malu Castellanos, Alkis Simitsis, Kevin Wilkinson, "Data Integraion Flows for Business Intelligence", ACM 978-1-60558422-5/09/0003, March 24-26, 2009.
[23] A White Paper, "Aggregation and analytics on Big Data using the Hadoop eco- system",2013.

[24] A White Paper, "SAAS Institute in USA, "Big Data Meets Big Data Analytics",2013.

[25] C.N.Hofer and G.Karagiannis, "Cloud Computing services: taxonomy and Comparison",2011.

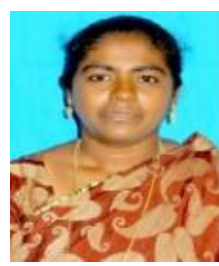

B. Arputhamary working as Assistant Professor in the Department of Computer Applications, Bishop Heber College, Tiruchirappalli, TamilNadu, India. She has 10 years of experience in teaching and 4 years of experience in research. Her areas of interests are: Cloud Computing, Big Data Analytics, Data Mining and Computer Networks. At present she is pursuing Ph.D., in Computer Science in Mother Teresa Women's University, Kodaikanal, Tamil Nadu.

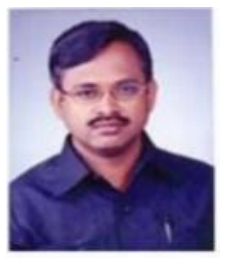

Dr. L. Arockiam is working as Associate Professor in the Department of Computer Science, St. Joseph's College, Tiruchirappalli, TamilNadu, India. He has 26 years of experience in teaching and 19 years of experience in research. He has published more than 213 research articles in the International \& National Conferences and Journals. He has also presented 3 research articles in the Software Measurement European Forum in Rome, Indonesia and Malaysia respectively. He is also the Member of IEEE, Madras Section and lifetime senior member of ISRD, London. He has chaired many technical sessions and delivered invited talks in National and International Conferences. He has authored 4 books. His research interests are: Cloud Computing, Big Data, Cognitive Aspects in Programming, Data Mining and Mobile Networks. He has been awarded "Best Research Publications in Science" for 2009, 2010 \& 2011 and ASDF Global "Best Academic Researcher" Award from ASDF, Pondicherry for the academic year 2012-13 and also the "Best Teacher in College" award for the year 2013 \& 2014. 